\title{
The Impact of a Drug Safety Warning on Discussions between Doctors and Their Patients; the Case of Rosiglitazone
}

\author{
Jim Nuovo
}

Department of Family \& Community Medicine, University of California, Davis, United States. Email: james.nuovo@ucdmc.ucdavis.edu

Received April $7^{\text {th }}$, 2011; revised June 20 ${ }^{\text {th }}, 2012$; accepted June 30 2011.

\begin{abstract}
The goal of this study was to track the influence of a highly publicized report on discussions between doctors and their patients and prescribing decisions made in response to concerns about potential medication adverse side effects. This was a retrospective analysis of a primary care network's electronic medical record database. From a diabetes registry of 12, 246 patients, 329 were identified as taking rosiglitazone prior to the June 14, 2007 release of an article in the New England Journal of Medicine; the article suggesting an increased risk of myocardial events. The entire content of all office visits, telephone messages, and medication lists for each patient were reviewed over a 2-year period subsequent to the article's publication. Doctor/patient discussions regarding concerns for rosiglitazone were catalogued including the physician's treatment recommendations. There were documented discussions on rosiglitazone's potential adverse side effects for 64 patients; 19.5 percent of this population. All of the discussions occurred between June 15 and October 30, 2007. Of the entire group, 59.3 percent $(N=195)$ remained on rosiglitazone. For those advised to continue rosiglitazone, the provider indicated that he/she wanted more data before determining if the drug was not safe or discounted the validity of the safety concerns. For those advised to discontinue rosiglitazone, 112 (83.6 percent) were placed on pioglitazone. An article suggesting potential adverse effects of rosiglitazone resulted in a documented discussion in 19.5 percent of patients on this medication. These findings suggest an awareness of this publication by patients, presumably derived from media reports. However, an awareness of this concern did not result in a substantial change in practice. The majority of patients remained on rosiglitazone. The content of these discussions suggest that most physicians' recommended waiting for more published data before considering a change. While many factors influence physician's prescribing behavior, this study demonstrates how a highly publicized report influences the doctor/ patient dialogue.
\end{abstract}

Keywords: Drug Safety, Patient-Physician Relationship, Rosiglitazone

\section{Introduction}

Thiazolidinediones (TZDs) have been recommended as part of the treatment plan for patients with Type 2 diabetes (DM). Rosiglitazone and pioglitazone are currently approved in most countries either as monotherapy, in combination with sulfonylureas or metformin, or in combination with insulin. In comparison to sulfonylureas and metformin, TZDs have a modest impact in decreasing glycosylated hemoglobin (A1C); the mean decrease in $\mathrm{A} 1 \mathrm{C}$ ranging from $0.7 \%$ to $1.6 \%$. Common adverse side effects from TZDs include weight gain, fluid retention, and edema. Serious side effects have been reported and include hepatotoxicity and congestive heart failure
[1]. On June 14, 2007 the New England Journal of Medicine published a report on the potential adverse side effects of rosiglitazone. The key finding of this article was as follows: "Rosiglitazone was associated with a significant increase in the risk of myocardial infarction and with an increase in the risk of death from cardiovascular causes that had borderline significance" [2].

Reports of potential harm from medications can be disseminated rapidly from the index researcher, through the scientific literature, media channels and then to physicians and the general public [3-5]. For specific medications, the impact of these reports is well-documented and may have a substantial effect on physician prescribing behavior. Notable examples of this have included the 
impact of trials on utilization of hormone replacement therapy (HRT) and cyclooxygenase-2 (COX-2) inhibitors [3-5]. How physicians perceive the effect of a drug warning published in the medical literature, what actions they take and how patients respond to these concerns has not been well studied. There are few studies describing the impact of a drug warning on the dialogue between doctors and their patients and the subsequent treatment decisions. Tracking discussions between doctors and their patients and the actions taken in response to these concerns can add to our understanding of what influences prescribing decisions. The purpose of this study was to describe the specific discussions between doctors and their patients that occurred after the dissemination of this New England Journal of Medicine report and the actions taken in response to the discussion.

\section{Methods}

A retrospective analysis was performed using the UC Davis Health System's (UCDHS) electronic medical record (EMR) database (Epic, Verona, WI). Patient data were obtained by querying the EMR database. This database has been previously validated for its accuracy in identifying all UCDHS patients with DM and in its ability to produce outcome-specific reports [6]. A search was conducted for all patients on avandia, avandamet, or rosiglitazone for the time period of June 1, 2006 through June 1, 2007. A report was generated with each patient's name and medical record number. The entire content of all office visits as well as phone messages, medication lists, medication orders were reviewed for one year prior to and two years after the New England Journal of Medicine report. Physician comments about rosiglitazone were cataloged verbatim along with the treatment plan; to continue the drug or to switch to another agent. Patients with no evidence of ongoing clinical activity within the past calendar year; i.e. no office visits, telephone messages, or evidence of any refill requests were not included in the study. This work was approved by the UC Davis Institutional Review Board.

\section{Results}

Summary of results from the electronic patient registry; those who were initially on rosiglitazone before the New England Journal of Medicine article, those who remained on it for the 2-year observation period, and those who were switched to pioglitazone is presented in Figure 1. There were 12,246 patients with DM treated at 13 UCDHS primary care clinics by 66 family physicians and general internists. There were 355 patients listed as

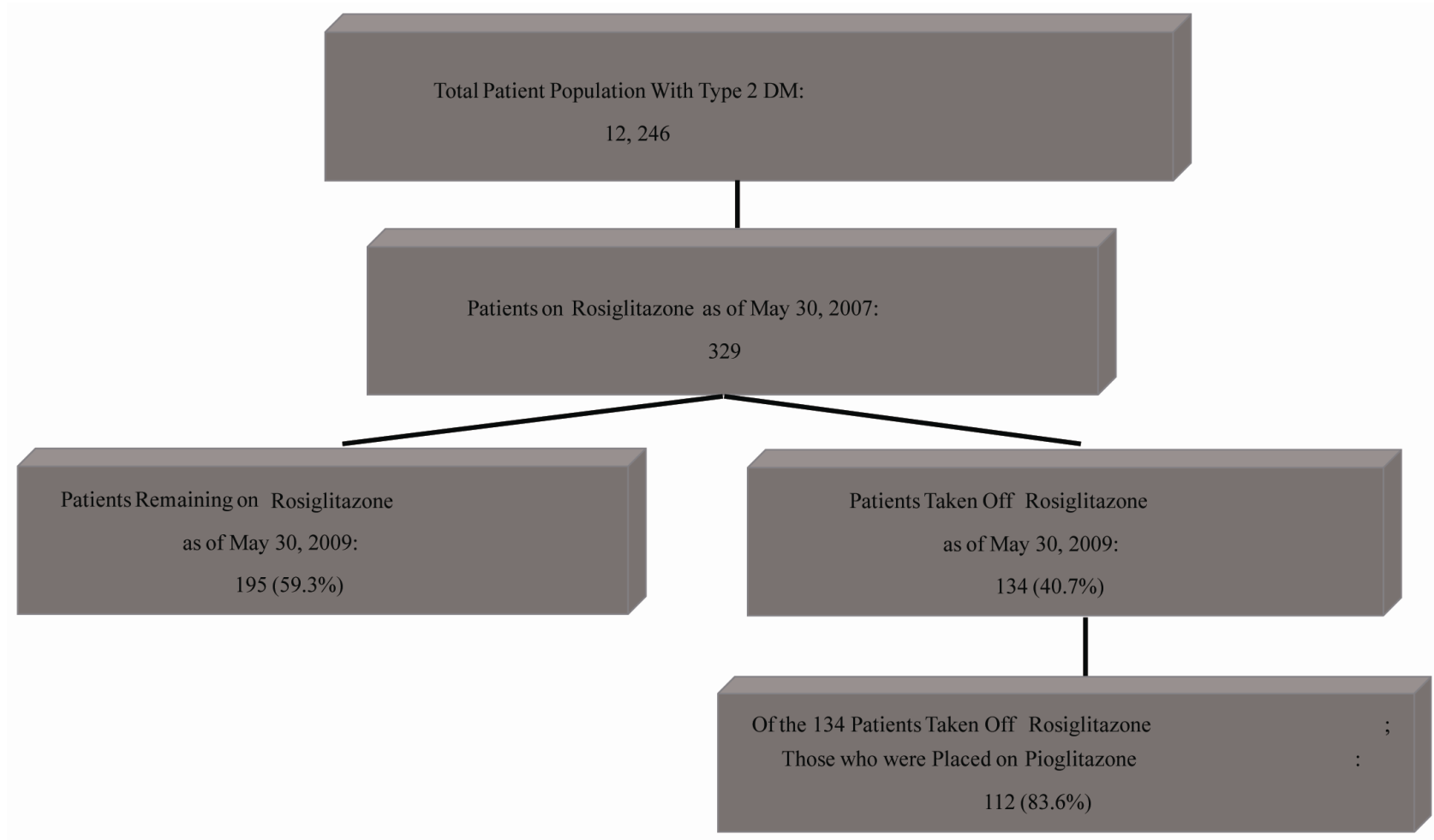

Figure 1. Summary of results from the electronic patient registry; those who were initially on rosiglitazone before the New England Journal of Medicine article, those who remained on it for the 2-year observation period, and those who were switched to pioglitazone. 
being on rosiglitazone on the May 30, 2007 registry report. Twenty-six of these patients had no evidence of ongoing clinical activity for the prior year and were removed from the study. There were 329 (2.7 percent of the total patient population) active patients on rosiglitazone as of this date. Of these 329 patients, 64 (19.5 percent) had documented subsequent discussions on rosiglitazone's potential adverse side effects. All of these discussions occurred between June 15 and October 30, 2007. Of the entire group, 195 patients (59.3 percent) remained on rosiglitazone. For those advised to continue rosiglitazone, the provider indicated that he/she wanted more data before determining if the drug was not safe. For those advised to discontinue rosiglitazone, 112 patients (83.6 percent) were placed on pioglitazone. Representative dyad comments documenting a dialogue between patients and their physician about safety concerns are presented in Table 1. All comments are extracted verbatim from the medical record. These comments are or- ganized into four categories based on whether there is a documented patient concern, physician response, and whether the rosiglitazone is or is not continued.

\section{Discussion}

This study explored the effects of a highly publicized report on the potential adverse side effects associated with rosiglitazone. There were two main purposes to this project: 1) To document the types of discussions between doctors and their patients, and 2) To assess whether there was any change in prescribing.

There was documentation in the medical records in 19.5 percent of patients on rosiglitazone. All of these discussions occurred within an 18-week period. For the 59.3 percent of patients advised to continue rosiglitazone, two themes emerged from the documented comments. First, the provider wanted more data before determining if the drug was not safe. Second, the provider discounted the validity of the New England Journal of Medicine

Table 1. Representative dyad comments documenting a dialogue between patients and their physician about safety concerns. All comments are extracted verbatim from the medical record.

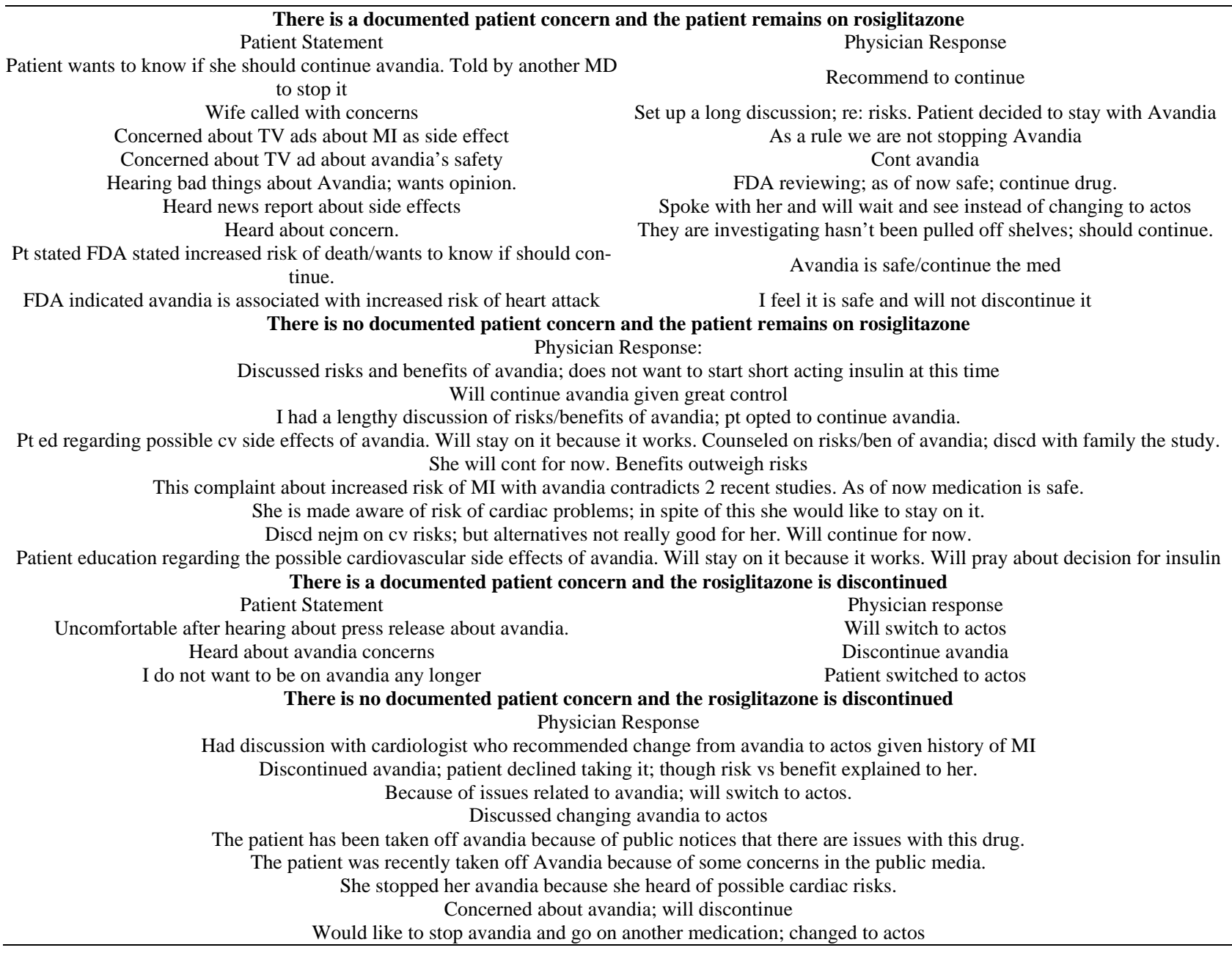


article. For the 40.7 percent who were switched to another medication, the most common theme abstracted from the comments was that the patient was concerned about the reports and preferred to make the change.

The timeframe of response and decline in use of rosiglitazone in this study is similar to that reported in studies of other medications after reports of concerning adverse side effects. Previous work on reports of adverse events related to calcium channel blockers in the setting of myocardial infarction demonstrated a 20 percent reduction of the use of this class of medications within months of the published report [7]. A similarly rapid, but larger reduction (66 percent) in the use of Prempro was noted over a 6-month period after reports of increased risk of breast cancer and cardiovascular disease were linked with estrogen/progestin combination therapy [3].

Another important finding from this study is how physicians' perceive a drug side effect as being unique to the drug and not class specific. The majority of patients who were changed to another medication were placed on pioglitazone. Given this information, it seems likely that many providers felt that this is not a class effect. This finding is supported by a recent study by Cohen and associates [8]. They found that while rosiglitazone use declined sharply from 0.42 million monthly treatment visits (February 2007) to 0.13 million monthly treatment visits (May 2008); pioglitazone use remained stable. Given that the FDA advisory was "class-level" for these agents, it is unclear how physicians came to make the distinction between rosiglitazone and pioglitazone.

Following the patients over a 2-year period demonstrated that there was little change over time for those who were advised to continue rosiglitazone. However, there were few new starts of rosiglitazone. Only 4 patients on the registry were started on this drug during the two-year period observation period; from June 14, 2007 through June 14, 2009. Again, this is consistent with the findings of Cohen and associates [8].

This study has a number of limitations. The information was abstracted from the medical records and relied on documentation. It is likely that this led to underreporting of discussions between doctors and their patients. This study cannot reliably determine the source of information which led to the discussion. Some of the abstracted comments attributed to patients' suggest that concerns solely came from the newsmedia; however, there may have been other sources that prompted patients to express their concerns; e.g. family members, pharmacists, and the internet. This study cannot determine the extent of concern exhibited by the patient to the provider and the extent to which that influenced the physician's response. Further, there may have been other characteristics of patients such as age, gender, ethnicity, socioeco- nomic status, level of diabetes control, and prior treatments which could have influenced the physician's response to these concerns. Length of use may also play a role. Patients on rosiglitazone for longer periods of time with no adverse side effects may perceive their risk for harm differently. This response was described by Faber and associates in their study on the influence of studies on harm associated with HRT [9]. Specifically, longterm HRT users were less likely to discontinue their medication. This study could not determine the role of style differences among physicians in the outcome of this study. While all providers were primary care physicians; i.e. family physicians and general internists; it is likely that these physicians view risk and assess the literature in a different manner or are more strongly influenced by other sources; e.g. opinions of colleagues, specialists, or pharmaceutical representatives. Again, this study could not determine how the decision was finally made. It appears that many patient concerns were met with recommendations to continue the drug.

This study also provides important lessons learned for future research on the practice effects when faced with the report of potential harm. There is a need for more research on the factors which influence decision-making and the role of the patient in this process. For example, the documented comments suggest that patients were the ones who initiated the discussion about adverse side effects with their providers in many of these cases. However, the majority of patients remained on rosiglitazone. This finding is somewhat contrary to the findings of prior research on how patients influence physicians. Kravitz and associates demonstrated that patients may have a substantial influence on physicians prescribing behavior [10]. In this community-based randomized trail, antidepressants were prescribed far more often when standardized patients requested them. This might potentially mean that there is a different perception among physicians in starting versus discontinuing a medication.

Future research should also consider the influence of the media; including the journal source. High impact journals are perceived as having more influence on providers. While the impact factor was not intended to be a marker for likelihood of practice change, this study brings into focus the question of what sources a physician trusts when concerns for harm are reported and warrants further investigation [11]. The expansion in use of social media Web sites, the credibility of this information, and how it may influence doctor/patient discussions also warrants further investigation.

Finally, future research should assess the influence of consensus statements by specialty organizations and warnings by the Food and Drug Administration (FDA). For example, despite statements from the American Di- 
abetes Association and the European Association for the Study of Diabetes recommending against the drug's use, data showed that use persisted [12]. After the FDA boxed warning was issued, there remained substantial use of rosiglitazone in certain geographic areas of this country [13].

In conclusion, the majority of physicians in this study continued to prescribe rosiglitazone after a highly publicized report demonstrated possible harm from the drug. Physician's documented comments suggest that they either: wanted more data before determining if the drug was not safe or discounted the validity of the safety concerns. Further research into the factors that influence patients and physician's when a report of harm is published is warranted.

\section{References}

[1] H. Yki-Jarvinen, "Thiazolidinediones," The New England Journal of Medicine, Vol. 351, 2004, pp. 1106-1118. doi:10.1056/NEJMra041001

[2] S. E. Nissen and K. Wolski, "Effect of Rosiglitazone on the Risk of Myocardial Infarction and Death from Cardiovascular Causes," The New England Journal of Medicine, Vol. 356, 2007, pp. 2457-2471. doi:10.1056/NEJMoa072761

[3] A. L. Hersh, M. J. Ste and R. S. Stafford, "National Use of Posmenopausal Hormone Thrapy: Annual Trends and Response to Recent Evidence,” The Journal of American Medical Association, Vol. 291, No. 1, 2004, pp. 47-53. doi:10.1001/jama.291.1.47

[4] S. R. Majumdar, E. A. Almasi and R. S. Stafford, "Promotion and Prescribing of Hormone Therapy after Report of Harm by the Women's Health Initiative,” The Journal of American Medical Association, Vol. 292, No. 16, 2004, pp. 1983-1988. doi:10.1001/jama.292.16.1983

[5] F.-Y. Hsiao, Y.-W. Tsai and W.-F. Huang, "Changes in Physician's Practice of Prescribing Cyclooxygenase-2 Inhibitor after Market Withdrawal of Rofecoxib: A Retrospective Study of Physician-Patient Pairs in Taiwan," Clinical Therapeutics, Vol. 31, No. 11, 2009, pp. 2618-

\section{7. doi:10.1016/i.clinthera.2009.11.022}

[6] E. M. Geraghty, T. Balsbaugh, J. Nuovo, et al., "Using Geographic Information Systems (GIS) to Assess Outcome Disparities in Patients with Type 2 Diabetes and Hyperlipidemia," The Journal of American Board of Family Medicine, Vol. 23, No. 1, 2010, pp. 88-96. doi:10.3122/jabfm.2010.01.090149

[7] S. R. Majumdar, T. S. Inui, J. H. Gurwitz, et al., "Influence of Physician Specialty on Adoption and Relinquishment of Calcium Channel Blockers and Other Treatments for Myocardial Infarction,” Journal of General Internal Medicine, Vol. 16, No. 6, 2001, pp. 351359. doi:10.3122/jabfm.2010.01.090149

[8] A. Cohen, A. Rabbani, N. Shah, et al., "Changes in Glitazone Use among Office-Based Physicians in the U.S., 2003-2009,” Diabetes Care, Vol. 33, No. 4, 2010, pp. 823-825. doi:10.2337/dc09-1834

[9] A. Faber, M. L. Bouvy, L. Loskamp, et al., "Dramatic Change in Prescribing of Hormone Replacement Therapy in the Netherlands after Publication of the Million Women Study: A Follow-Up Study,” British Journal of Clinical Pharmacology, Vol. 60, No. 6, pp. 641- 647.

[10] R. L. Kravitz, R. M. Epstein, M. D. Feldman, et al., "Influence of Patient's Requests for Direct-to-Consumer Advertised Antidepressants: A Randomized Controlled Trial," The Journal of the American Medical Association, Vol. 293, No. 16, 2005, pp. 1995-2002. doi:10.2337/dc09-1834

[11] E. Garfield, "The History and Meaning of the Journal Impact Factor," The Journal of the American Association, Vol. 295, No. 1, 2006, pp. 90-93. doi:10.2337/dc09-1834

[12] J. A. Greene and A. S. Kesselheim, "Pharmaceutical Marketing and the New Social Media," The New England Journal of Medicine, Vol. 363, 2010, pp. 2087-2089. doi:10.1056/NEJMp1004986

[13] N. D. Shah, V. M. Montori, H. M. Krumholz, et al., "Responding to an FDA Warning-Geographic Variation in the Use of Rosiglitazone," The New England Journal of Medicine, Vol. 363, 2010, pp. 2081-2084. doi:10.1056/NEJMp1011042 